\title{
Terry's Nails: Clinical Correlations in Adult Outpatients
}

\author{
Nick Nelson, MBBS ${ }^{7}$, Kweku Hayfron, $\mathrm{MD}^{2}$, Alejandro Diaz, MD ${ }^{7}$, Scott Lynch, MD' , Irene Yen, PhD ${ }^{3}$, \\ Allison Bakamiian, MPH, MSW', and Indhu Subramanian, $\mathrm{MD}^{7}$ \\ 'Wing A2 Department of Medicine Annex, Highland Hospital, Oakland, CA, USA; ${ }^{2}$ Sutter Health/Palo Alto Medical Foundation, Dublin, CA, USA; \\ ${ }^{3}$ Highland Hospital, UC San Francisco, San Francisco, CA, USA; ${ }^{H}$ Highland Hospital, UC Berkeley, Berkeley, CA, USA.
} KEY WORDS: diagnosis; gastroenterology; primary care; physical
examination; cirrhosis.

J Gen Intern Med 33(7):1018-9

DOI: $10.1007 / \mathrm{s} 11606-018-4441-7$

(C) Society of General Internal Medicine 2018

\section{INTRODUCTION}

The nails have a rich history as windows into systemic disease, beginning with Hippocrates' observation of digital clubbing as a sign of empyema in the 5 th century BCE. ${ }^{1}$ In 1954 , Richard Terry added to this tradition the sign which bears his name when he reported "a ground-glass-like opacity of almost the entire nail bed". ${ }^{2}$ He observed this abnormality most frequently in patients with cirrhosis, but also in cases of congestive heart failure (CHF), diabetes, tuberculosis, rheumatoid arthritis, systemic sclerosis, some cancers, and in normal, healthy children up to age four. ${ }^{21}$ Terry's nails have also been reported in reactive arthritis, renal transplant patients, POEMS syndrome, and leprosy, and have recently been promoted as one of the most reliable physical signs of cirrhosis $^{3}$ (Fig. 1).

The only systematic study of Terry's nails in English was conducted on medical inpatients and confirmed, using slightly modified criteria, associations with cirrhosis, CHF, and adultonset diabetes, ${ }^{4}$ findings which were later corroborated by a similar observational study of inpatients. ${ }^{5}$

The prevalence and clinical significance of Terry's nails has never been systematically studied in outpatients. We sought to determine the prevalence and clinical correlates of Terry's nails in a sample of adults recruited from a general medicine clinic at a community teaching hospital.

\section{METHODS}

We conducted a prospective, cross-sectional observational study. We photographed the fingers and toes of a consecutive sample of 503 outpatients. Photographs were obtained using a standardized procedure and analyzed by four physicians who had been trained to recognize Terry's nails according to the

'This finding was anecdotally confirmed in two of the authors' newborn children during the study period (NN and SL, $N=2$ ).

Published online April 17, 2018 modified criteria of Holzberg and Walker, ${ }^{4}$ with images drawn from peer-reviewed case reports serving as the standard of reference. Each reviewer examined all images, and for each patient recorded whether Terry's nails were present, absent, or indeterminate. The analysts were blinded to one another and to the patient's medical history.

Research assistants blinded to the photographs reviewed medical records according to a protocol to determine the presence or absence of conditions associated with Terry's nails. Datasets were then merged to establish the interobserver agreement of the analysts, the prevalence of Terry's nails in the sample, and the sign's clinical correlations.

\section{RESULTS}

The inter-observer reliability of the four raters for Terry's nails observed in the fingers was $\kappa=0.56$, indicating moderate agreement.

We observed no association between Terry's nails of the fingers or toes and CHF, diabetes, hepatitis $\mathrm{C}$, chronic kidney disease, hypoalbuminemia, or elevated aminotransferase levels.

The only clinically significant association observed was between Terry's nails in the fingers and the presence of cirrhosis, with a positive likelihood ratio of 3.7 to 10.1, depending on the observer (Table 1).

\section{DISCUSSION}

This study sought to determine the clinical diagnostic utility of Terry's nails in a convenience sample of adult outpatients in a general medicine clinic. We were unable to demonstrate associations previously reported with CHF and diabetes, but confirmed a strong association with cirrhosis.

Our study had several limitations. First, data on disease status was not available for all patients. This may have led to under-diagnosis of the conditions of interest, reducing our ability to detect associations. Second, the prevalence of Terry's nails was fivefold less in our sample than in Holzberg and Walker's (3-8\% vs. $25.2 \%){ }^{4}$ Nonetheless, our study accurately reflects the conditions of outpatient practice in general medicine, and we believe that a few conclusions are possible. 


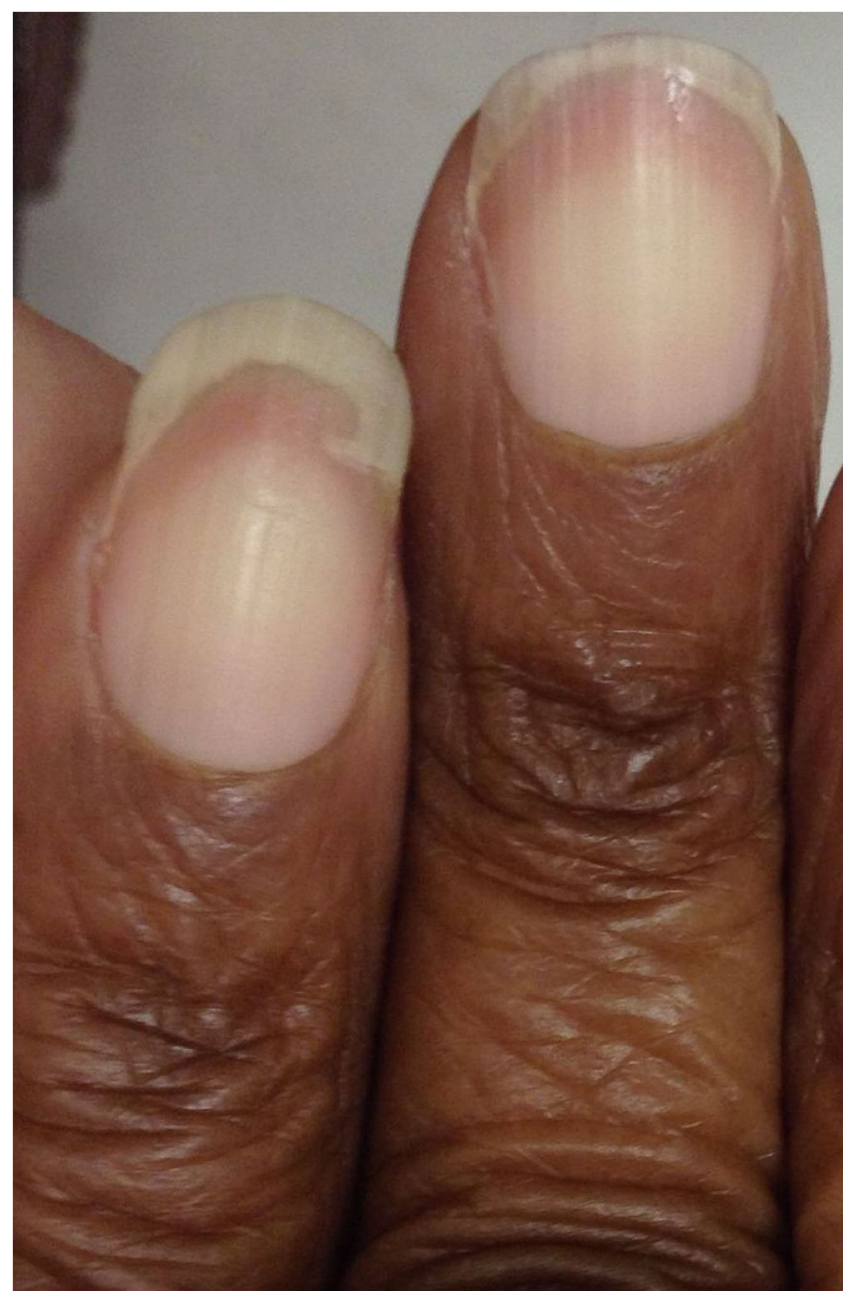

Figure 1 Terry's nails

First, Terry's nails appear to be about ten times less common among outpatients than inpatients, which suggests that their appearance is correlated with disease severity. Second, among outpatients, the sign is insensitive but highly specific for cirrhosis, and this is its only strong diagnostic association.
Table 1 Sensitivity, Specificity, and Likelihood Ratio of Terry's Nails for the Presence of Cirrhosis by Rater with $95 \%$ Confidence Interval

\begin{tabular}{llll}
\hline \hline Rater & Sensitivity & Specificity & +ve LR \\
\hline $\begin{array}{l}\text { Rater I } \\
\text { KH, PGY3) }\end{array}$ & $0.25(0.06-57.2)$ & $0.95(0.93-0.97)$ & $5.4(1.8-18.8)$ \\
$\begin{array}{l}\text { Rater II } \\
\text { (SL, PGY4) }\end{array}$ & $0.29(0.84-0.58)$ & $0.92(0.89-0.95)$ & $3.7(1.5-9.0)$ \\
$\begin{array}{l}\text { Rater III } \\
\text { (AD, PGY4) }\end{array}$ & $0.21(0.47-0.5)$ & $0.98(0.96-0.99)$ & $10.1(3.0-33.4)$ \\
$\begin{array}{l}\text { Rater IV } \\
\text { NN, PGY8) }\end{array}$ & $0.20(0.43-0.48)$ & $0.98(0.96-0.99)$ & $7.9(2.45-25.3)$ \\
\hline
\end{tabular}

Therefore, we conclude that clinicians observing Terry's nails in an unselected adult outpatient should specifically investigate the possibility of cirrhosis. Based on our results, it appears that the other reported associations are, at least in the outpatient context, too weak to warrant further testing absent other suggestive clinical features.

Corresponding Author: Nick Nelson, MBBS; Wing A2 Department of Medicine Annex, Highland Hospital, 1411 E 31st St., Oakland, CA 94602, USA (e-mail: nnelson@alamedahealthsystem.org).

\section{Compliance with Ethical Standards:}

Conflicts of Interest: The authors declare that they have do not have a conflict of interest.

\section{REFERENCES}

1. Hippocrates; Ducachet HW. The Prognostics and Crises, London: James Eastburn and Co.; 1819: 51.

2. Terry R. White nails in hepatic cirrhosis. Lancet. 1954; 266:757-759

3. Udell JA et al. Does this patient with liver disease have cirrhosis? JAMA. 2012; 307:832-842

4. Holzberg M, Walker HK. Terry's nails: revised definition and new correlations. Lancet. 1984;1:896-99.

5. Park KY, Kim SW, Cho JS. Research on the frequency of terry's nail in the medical inpatients with chronic illnesses. Korean J Dermatol. 1992; 30: 864-70. 